\title{
Temporal compatibility in dual motor tasks II: Simultaneous articulation and hand movements
}

\author{
STUART T. KLAPP \\ California State University, Hayward, California 94542
}

\begin{abstract}
Simultaneous articulation did not interfere with hand responses if the responses shared the same time period. Otherwise there was marked interference even after performance had stabilized with practice. These results generalized previous findings for simultaneous leftand right-hand responses (Klapp, 1979) to show that even highly distinctive responses can be generated in parallel only if they have compatible timing.
\end{abstract}

Simultaneous movements of the left and right hands interfere with each other more if they differ in temporal rhythm compared with simultaneous movements with the same or harmonically related rhy thms (Cohen, 1970; Klapp, 1979). Similarly, two hand tasks for which movements of differing temporal patterning would optimize performance tend to be made or programmed with the same timing rather than with the optimal different time patterns (Kelso, Southard, \& Goodman, 1979; Klapp \& Greim, 1979). ${ }^{1}$ These results suggest a "tendency of the nervous system to generate only one temporal pattern at a time," possibly due to the "wealth of interconnections within the system" (Klapp, 1979, p. 380). However, the presently available findings concern movements of the two hands, and therefore this single rhythm limitation might apply only to corresponding muscles on the two sides of the body or to muscles which are difficult to distinguish in some sense. ${ }^{2}$ The present experiments were designed to determine if the single rhythm limitation would generalize to simultaneous movements, which are more distinctive. Simultaneous hand tapping and articulation were selected as meeting this criterion.

\section{EXPERIMENT 1}

\begin{abstract}
Method
For all conditions the primary response was to press a standard telegraph key with the fingers of the right hand such that the key would be down when a $300-\mathrm{Hz}$ tone sounded in the right ear and up when the tone was not sounded. Presentation of the tone was repetitive at $300 \mathrm{msec}$ on and $500 \mathrm{msec}$ off. A 3,000-Hz alarm sounded in the left ear if the telegraph key was depressed during a 200-msec interval centered within the tone-off portion of the cycle or if the key was not depressed during a $100-\mathrm{msec}$ interval centered within the tone-on portion of the cycle. For each trial the tones were activated for 5 to $10 \mathrm{sec}$ before mea-
\end{abstract}

The author wishes to thank Eleazar Mora, who supervised collection of the data, and Ken Preston, who assisted him. This research was supported in part by the Small Grants Program of the Research Foundation of California State University, Hayward. Requests for reprints should be sent to Stuart T. Klapp, Department of Psychology, California State University, Hayward, California 94542. surements were taken. Then the number of alarm soundings was recorded for 20 consecutive cycles. The measured portion was followed by approximately $5 \mathrm{sec}$ of unmeasured performance. Thus each trial lasted about $30 \mathrm{sec}$, with data recorded only during the central portion of the trial to avoid start-up and terminal effects.

All of the three conditions involved the same primary response as described above, but differed with respect to the requirement of a concurrent articulatory response. For the press-only control condition there was no concurrent response. For the synchronized conditions subjects were to pronounce the syllable "la" at the same time that they pressed the telegraph key. For the fast-as-possible condition, subjects were to pronounce "la" continuously as rapidly as possible throughout each trial. These conditions are analogous to a previous test involving responses of the two hands (Klapp, 1979, Experiment 1).

Each of 12 subjects was tested in all three conditions, with the order of conditions balanced across subjects. Each condition was presented for 10 consecutive trials, with an intertrial interval of approximately $15 \mathrm{sec}$. The subjects were right-handed students at California State University, Hayward, who participated as one option in a course requirement in introductory psychology. Prior to any of the scored trials, subjects practiced all conditions in the order in which the scored conditions would later appear.

\section{Results and Discussion}

The mean number of alarm soundings per trial (20 cycles) differed among the three conditions $[F(2,22)$ $=28.7, \mathrm{p}<.001]$, as indicated in Table 1 . The comparisons involving two conditions were all significant [each $\mathrm{F}(1,11)>6.7, \mathrm{p}<.025]$. Clearly, responses which operate on the same temporal period (synchronized condition) interfere with each other less than do responses which operate on different periods (fast as possible). These results replicate the previous results (Klapp, 1979, Experiment 1) for two-hand responses, extending the findings to combined articulation and

Table 1

Hand Response Error Alarms Per Trial in Experiment 1

\begin{tabular}{lrrr} 
& \multicolumn{3}{c}{ Trials } \\
\cline { 2 - 3 } \multicolumn{1}{c}{ Condition } & $1-5$ & $6-10$ & Mean \\
\hline Control & 2.13 & 2.52 & 2.32 \\
Synchronized & 6.48 & 4.20 & 5.34 \\
Fast as Possible & 12.10 & 12.30 & 12.20 \\
\hline
\end{tabular}


hand responses. However, there is some ambiguity concerning whether the synchronized movements interfere relative to a single-hand movement control. Although significant, the decrement in performance for synchronized movements compared with the single-task control became smaller for later trials in the 10 trial blocks than for earlier trials, yielding a significant interaction $[F(9,99)=2.6, p<.025]$. For the two-hand case (Klapp, 1979), there was only a nonsignificant trend. suggesting that synchronized movements might interfere compared with a single movement control.

\section{EXPERIMENT 2}

A possible problem of interpretation of Experiment 1 is that the sound of the subjects' pronunciation might have interfered with hearing the tone and alarm signals controlling the primary response. Thus, the difference among conditions might be attributable to auditory interference rather than to response interference. Experiment 2 replicated Experiment 1 using unvoiced rather than voiced articulation.

\section{Method}

The method was identical to Experiment 1 except that the articulatory response was unvoiced to prevent auditory interference. Only six subjects were tested.

\section{Results and Discussion}

As in Experiment 1, the error alarm sounded more often for the fast-as-possible condition (mean 11.6/trial) than for either the synchronized condition $(7.7)[\mathrm{F}(1,5)$ $=15.0, \mathrm{p}<.025]$ or the press-only control condition (3.8) $[F(1,5)=25.5, p<.005]$. These findings replicate the results of Experiment 1 and indicate that responses which operate on the same temporal period (synchronized condition) interfere with each other less than do responses on different time periods (fast as possible). Since the articulation was not voiced in Experiment 2, auditory interference cannot account for this result.

Again, as in Experiment 1, there is some ambiguity involving the comparison between control and synchronized conditions. In Experiment 2 the difference was nonsignificant due to variations between subjects $[F(1,5)=3.9, p \approx .10]$. In Experiment 1 the difference tended to vanish with practice, but was significant overall.

\section{EXPERIMENT 3}

Experiments 1 and 2 showed more interference between rapid articulation and keypressing compared with the interference when articulation was synchronized with the keypress. This effect could be attributed either to the role of inconsistent rather than consistent timing of the two responses or to the rate of articulation. The advantage of using the fast-as-possible procedure of Experiments 1 and 2 was that the articulation response could occur without stimulus guidance. However, this experimental advantage had its cost in the confounding of response rate with temporal relations. Experiment 3 removed the confounding but required stimulus guidance for both the keypress and articulation responses. Testing was carried out over eight sessions per subject to determine whether performance for the various conditions might converge with practice. The general design of Experiment 3 was similar to a previous experiment using two-hand responses (Klapp, 1979, Experiment 4).

\section{Method}

For all conditions the right-hand task was similar to that of Experiments 1 and 2, except that the temporal period was lengthened to $1.5 \mathrm{sec}$ (compared with $800 \mathrm{msec}$ in Experiments 1 and 2). As before, the $300-\mathrm{Hz}$ signal tone sounded for $300 \mathrm{msec}$ in the right ear, and the subject was to press the telegraph key when the tone sounded. A $3,000-\mathrm{Hz}$ alarm sounded in the left ear if the telegraph key was depressed during a $900-\mathrm{msec}$ interval centered within the $1.2-\mathrm{sec}$ tone-off portion of the cycle, or if the key was not depressed during a 100-msec interval centered within the $300-\mathrm{msec}$ tone-on portion of the cycle. As in the previous experiment, the tones were activated for each trial for 5 to $10 \mathrm{sec}$ before measurements were taken and then the number of alarm soundings was recorded for 20 consecutive cycles of tone on/off. The trial then continued for an additional 5 or $10 \mathrm{sec}$ of unmeasured performance. Each trial lasted for approximately $45 \mathrm{sec}$.

The four conditions differed with respect to the type of silent articulation required concurrent with the handpressing task. In the control condition there was no articulation and no stimulus other than the previously mentioned signal and alarm tones. In the other three conditions articulation was signaled by a light that was displayed for $300 \mathrm{msec}$. The subject was to initiate a silent articulation of "la" at the onset of the light. The three conditions with articulation differed with respect to the timing of the light signal in relation to the tone signal. In the simultaneous condition the light was synchronized with the tone signal. In the same-period condition the light and tone signals appeared with the same temporal period $(1.5 \mathrm{sec})$ but out of phase. The light led the tone by $600 \mathrm{msec}$, so that light offset and tone onset were separated by $300 \mathrm{msec}$. In the differentperiod condition the light and tone signal had periods that were not harmonically related. There were two subconditions: one with a light signal period of $1.3 \mathrm{sec}$ (shorter period than the tone) and one with a period of $1.7 \mathrm{sec}$ (longer period than the tone). Half of the subjects received one subcondition and the remaining subjects received the other.

Each subject was tested in all four conditions, with the order of testing balanced by two different Latin squares: One Latin square applied to each subcondition of the different-period condition. Each condition was tested for a block of 10 consecutive trials. Four such blocks, one block for each condition, comprised a session. Each subject was tested for eight sessions, with the order of conditions constant across sessions for each subject (but balanced across subjects). Total testing time for the eight sessions was approximately $5 \mathrm{~h}$, spread over three meetings. The eight subjects were from the same population as in the previous experiments. One subject was replaced because she quit before testing was completed, complaining that the task was too difficult.

\section{Results and Discussion}

Performance differed among the four conditions $[F(3,21)=54.2, \mathrm{p}<.001]$ and improved with practice $[F(7,49)=2.4, p<.05]$ (see Figure 1). There was a 


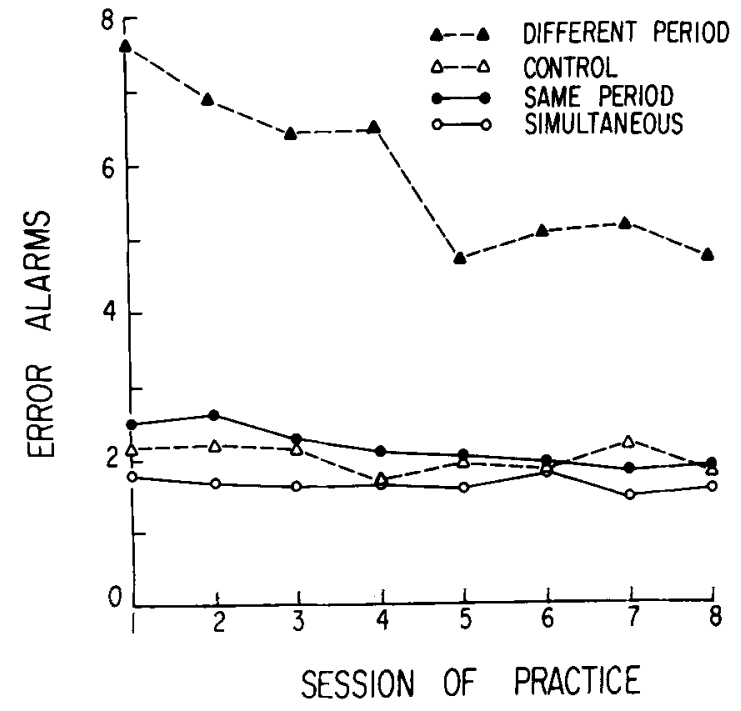

Figure 1. Hand response error alarms as a function of concurrent articulation and session of practice in Experiment 3.

marked Condition by Practice interaction $[F(21,147)=$ $5.0, p<.001]$, such that the difference among conditions decreased as practice progressed. However, inspection of Figure 1 suggests that both improvement with practice and the Practice by Condition interaction were confined to the first five sessions of practice. Over the last three sessions there was neither a significant effect of practice $[F(7,14)<1]$ nor any interaction involving practice $[F(21,42)<1]$. This result was similar to the previous report for two-hand responses (Klapp, 1979) in the stabilization of performance after five sessions of practice. The remaining analyses emphasize the last three sessions after performance had stabilized.

Even in the last three sessions, performance was much worse in the different-period condition than in any of the other three conditions [with $F(1,7)$ at least 30.2, $\mathrm{p}<.001$ ] for the comparisons. Performance did not differ significantly among the remaining three conditions $[F(2,14)=1.8]$. We conclude that two responses can be generated together if they have the same period with no interference compared with a single response control; responses with different temporal periods can be generated only with considerable interference.

The present results are in agreement generally with the corresponding results for two-hand responses (Klapp, 1979, Experiment 4), with one minor exception. In the two-hand case simultaneous (in-phase) responses interfered more than did out-of-phase responses of the same period. In the articulation/hand results of the present experiment there was no significant difference between these conditions $[F(1,7)=1.9, p>.20]$. The nonsignificant trend was opposite to the significant results of the previous experiment, and was significant over the first five sessions $[F(1,28)=10.9, p<.025]$. This suggests the possibility that in-phase responses (simultaneous) may interfere less than do out-of-phase (same-period) responses. For the two-hand results I suggested (Klapp, 1979, p. 380) that "performance may be degraded by the overlap of muscular activity." That statement can now be seen to apply for two-hand movements but not for hand movements coordinated with articulatory responses. Perhaps there is a special case of interference that occurs when people try to make identical responses with two hands that does not generalize to synchronized responses of different effectors. However, any such effect is weak in comparison with the effects of temporal compatibility.

\section{GENERAL DISCUSSION}

Rhythmic articulation interfered with simultaneous rhythmic hand movements if the two responses differed in timing. Much less interference occurred if the articulation and manual responses had the same temporal periods, even if they differed in phase. However, performance on articulation (unvoiced in Experiments 2 and 3) was not measured, raising the possibility that articulatory performance might have been improved by lack of temporal compatibility-a manipulation that reduced performance in the concurrent manual task. ${ }^{3}$ If this were the case, then the observed reduced manual performance could be attributed to allocation of resources to favor articulation rather than to interference between incompatible timings. This possibility seems unlikely, both because of the implausible assumption of improved performance with conflicting timing and because of a mutual reduction of performance was found in parallel experiments involving two manual responses (Klapp, 1979). We concluded that there is considerable difficulty in generating concurrent rhythmic articulatory and manual responses that are not temporally compatible in the sense of having the same period of repetition. This conclusion generalizes the previous results for responses of the left and right hand (Klapp, 1979) to show that the timing limit extends beyond homologous muscle groups such as those for the two hands.

A theoretical account of the single timing limit has been advanced by Kelso et al. (1979). In this view there is a "coordinative structure or muscle linkage" (Kelso et al., p. 236) that forms a functional group to perform a particular task. The difficulty in performing tasks that differ in timing is taken as one form of evidence for these groupings. Although this theory has proven useful in other contexts, it does not seem to account for the present phenomenon. The tasks under study can be better performed if the muscles are not coordinated in time; indeed, it is the required lack of coordination that makes these tasks difficult. However, one could argue that the two hands are frequently used together as a functional unit, and the functional grouping cannot be broken even when the task requires a lack of coordination. This type of argument could account for timing 
limits involving the hands, but it does not seem relevant for combined articulation and hand movements that do not tend to function together to perform a unified response, as is common with the two hands. Nevertheless, as we have demonstrated, people have considerable difficulty in uncoupling the timings of hands and articulators.

In our view (Klapp, 1979) the limitation of concurrent responses to a single time frame is due to the interconnections within the nervous system which may lock all responses into synchrony, even if the responses have no functional unity. The limit is not attributed to insufficient processing capacity. Presumably, two independent rhythms could be generated by two independent nervous systems of much less total processing capacity than that of our human subjects. Some support for our neural interconnection viewpoint is given by the observation that a patient who was allegedly suffering from a partial deconnection of the two cerebral hemispheres could generate movements with the two hands that differed in timing (Geshwind \& Kaplan, 1962).

An issue of emerging importance in the information processing analysis of motor control concerns the role of timing vs. muscular equilibrium (position) in the control of motor responses (Keele, 1981). Some theories stress timing (e.g., Klapp, 1977; Klapp \& Greim, 1979; Schmidt, Zelaznik, Hawkins, Frank, \& Quinn, 1979; Summers, 1977), but others do not (e.g., Mays \& Sparks, 1980; Schmidt, 1980). The present results indicate that a basic limitation of motor response generation concerns the necessity to combine all simultaneous responses into a single temporal frame. This conclusion suggests that a stress on timing in theoretical analysis of motor control is justified.

\section{REFERENCES}

CoHEN, L. Interaction between limbs during bimanual voluntary activity, Brain, 1970, 93, 259-272.

Duncan, J. Divided attention: The whole is more than the sum of its parts. Journal of Experimental Psychology: Human Perception and Performance, 1979, 5, 216-228.

Geschwind, N., \& Kaplan, E. A human cerebral deconnection syndrome. Neurology, 1962, 12, 675-685.

KEELE, S. W. Behavior analysis of movement. In V. Brooks (Ed.), Handbook of physiology. Bethesda, Md: American Physiological Society, 1981.

Kelso, J. A. S., Southard, R. L., \& Goodman, D. On the coordination of two-handed movements. Journal of Experimental Psychology: Human Perception and Performance, 1979, 5, 229-238.

KLAPP, S. T. Reaction time analysis of programmed control. In $\mathbf{R}$. Hutton (Ed.), Exercise and sports sciences reviews. Santa Barbara, Calif: Journal Publishing Affiliates, 1977.

KLAPP, S. T. Doing two things at once: The role of temporal compatibility. Memory \& Cognition, 1979, 7, 375-381.

Klapp, S. T., \& Greim, D. M. Programmed control of aimed movements revisited: The role of target visibility and symmetry. Journal of Experimental Psychology: Human Perception and Performance, 1979, 5, 509-521.

MAYs, L. E., \& Sparks, D. L. Saccades are spatially, not retinocentrically coded. Science, 1980, 208, 1163-1165.

Peters, M. Simultaneous performance of two motor activities: The factor of timing. Neuropsychologia, 1977, 15, 461-465.

Schmidt, R. A. Past and future issues in motor programming. Research Quarterly, 1980, 51, 122-140.

Schmidt, R. A., Zelaznik, H. N., Hawkins, B., Frank, J. S., \& QUiNN, J. T. JR. Motor-output variability: A theory for the accuracy of rapid motor acts. Psychological Review, 1979 , 96, 415-451.

Summers, J. J. The relationship between the sequencing and timing components of a skill. Journal of Motor Behavior, 1977, 9, 49-59.

\section{NOTES}

1. There is also a tendency to generate the same timing even though the task demands harmonically related timing for two hands (Duncan, 1979).

2. Peters (1977, Experiments 2 and 3) reports an informal observation that "very few subjects were able to perform" the task of generating incompatible two-hand or hand/voice rhythms.

3 . Between-condition comparisons of articulatory performance would be meaningful only when the articulations compared are the same except in temporal relation to the hand response.

(Received for publication July 14, 1980; revision accepted February 17, 1981.) 